ISSN: 0210-1696

DOI: http://dx.doi.org/10.14201/scero20174822540

\title{
EFECTOS Y BENEFICIOS \\ DE LA PLANIFICACIÓN CENTRADA \\ EN LA PERSONA (PCP) EN LAS PERSONAS \\ ADULTAS CON DISCAPACIDAD \\ INTELECTUAL, EN LAS ORGANIZACIONES \\ Y EN LA COMUNIDAD. ESTADO \\ DE LA CUESTIÓN
}

Effects and benefits of person-centered planning

$(P C P)$ in adults with intellectual disabilities, in organizations and in community. State of the art

Tania Cuervo Rodríguez

Universidad de Oviedo. Departamento de Ciencias de la Educación

C/ Aniceto Sela, s/n. 33005 Oviedo

UO194650@uniovi.es

María Teresa IgLEsias García

Universidad de Oviedo. Departamento de Ciencias de la Educación

Samuel FERNÁNDEZ FERNÁNDEZ

Universidad de Oviedo. Departamento de Ciencias de la Educación

Recepción: 20 de octubre de 2016

Aceptación definitiva: 8 de febrero de 2017

Resumen: Desde hace más de tres décadas se ha venido proponiendo la aplicación de una metodología denominada Planificación Centrada en la Persona (PCP), que parte de los principios de normalización e inclusión, y en la que la persona con discapacidad intelectual se convierte en el centro de toda la intervención, pasando de ser un mero 
EFECTOS Y BENEFICIOS DE LA PLANIFICACIÓN CENTRADA EN LA PERSONA (PCP) EN LAS PERSONAS ADULTAS CON DISCAPACIDAD INTELECTUAL, EN LAS ORGANIZACIONES Y EN LA COMUNIDAD

TANIA CUERVO RODRÍGUEZ, MARÍA TERESA IGLESIAS GARCÍA Y SAMUEL FERNÁNDEZ FERNÁNDEZ

receptor de servicios a ser un sujeto activo, con plenos derechos y al que se le deben prestar los apoyos necesarios para que pueda cumplir sus sueños, metas y/o deseos.

En este artículo se realiza una revisión bibliográfica cuya finalidad es analizar los beneficios de la aplicación de la PCP en las unidades y organismos que atienden a las personas con discapacidad. Para ello se ha realizado una amplia búsqueda de documentos en seis bases de datos de carácter nacional e internacional, utilizando los descriptores de "planificación centrada en la persona", "pcp”, "person centred planning” y "person centered planning”. Siguiendo una serie de criterios establecidos por los autores se han seleccionado 18 artículos a analizar. Los resultados muestran que la PCP produce cambios en las personas con discapacidad, en las organizaciones y en la comunidad, a pesar de existir una serie de barreras para su implementación, tal y como se describe en el artículo.

Palabras Clave: discapacidad intelectual; planificación centrada en la persona; PCP; cambio organizacional; revisión bibliográfica.

AвSTRACT: For more than three decades it has been developing a methodology called Person-Centered Planning (PCP), where the person with intellectual disability becomes the center of all planning, being a subject with rights and to who should be given the necessary support to fulfill his/her dreams, goals and/or desires. This article presents a literature review aimed to know the benefits of the implementation of the PCP in services that carer to people with disabilities. For it has been made an exhaustive research in 6 national and international databases, using descriptors "planificación centrada en la persona", "pcp", "person centred planning” and "person centered planning”. Following a series of criteria established by the authors we have selected 18 items to be analyzed. The results show that the PCP is a tool that produces changes in the person, in the organizations and in the community, despite there being a number of barriers to implementation as been explained throughout this article.

KEYWORDS: intellectual disability; person-centered planning; PCP; organizational change; literary review.

\section{Un acercamiento a la planificación centrada en la persona}

4 N LOS AÑOS 70 y 80 se produce un acercamiento ideológico a los principios de normalización e inclusión de las personas con discapacidad intelectual (Davis, Cornman, Lane y Patton, 2005; Claes, Hove, Vandevelde, Loon y Schalock, 2010) y, a su vez, se ha ido desarrollado un cambio en la concepción de la discapacidad, pasando de un modelo médico a un modelo de carácter más social (Pallisera, 2011). Fruto de ello se han podido apreciar varios avances en el campo del diagnóstico, tal como la Clasificación Internacional de Funcionamiento, de la Discapacidad y de la Salud (CIF), y en el campo metodológico, el desarrollo de la Planificación Centrada en la Persona (PCP de aquí en adelante) (Carbonell, 2006; Lunt y Hinz, 2014).

Aun habiendo pasado más de 3 décadas desde su introducción en las políticas de atención a las personas con discapacidad en determinados países como Reino Unido y Estados Unidos (Robertson, Emerson, Elliott y Hatton, 2008), sigue sin haber un

(C) Ediciones Universidad de Salamanca / CC BY-NC-ND

Siglo Cero, vol. 48 (2), n. ${ }^{\circ}$ 262, 2017, abril-junio, pp. 25-40 
EFECTOS Y BENEFICIOS DE LA PLANIFICACIÓN CENTRADA EN LA PERSONA (PCP) EN LAS PERSONAS ADULTAS CON DISCAPACIDAD INTELECTUAL, EN LAS ORGANIZACIONES Y EN LA COMUNIDAD TANIA CUERVO RODRÍGUEZ, MARÍA TERESA IGLESIAS GARCÍA Y SAMUEL FERNÁNDEZ FERNÁNDEZ

acuerdo entre los diferentes autores sobre cuál es la correcta definición de PCP (Flannery et al., 2000). En la Tabla 1 se muestran varias de las concepciones que han ido surgiendo a lo largo de los últimos años.

\begin{tabular}{|l|l|}
\hline \multicolumn{2}{|c|}{ TABLA 1. Definiciones sobre PCP } \\
\hline & \multicolumn{1}{|c|}{ DefinICIONES } \\
\hline $\begin{array}{l}\text { Cole, McIntosh } \\
\text { y Whittaker (2000) }\end{array}$ & $\begin{array}{l}\text { Es un proceso en el que se trata de conocer lo que la persona quiere conseguir con su vida, y } \\
\text { se le ayuda a decidir sobre los objetivos y luego se planea qué acciones deben realizarse para } \\
\text { conseguirlos. Es un proceso continuo, lo que significa que la persona puede cambiar perió- } \\
\text { dicamente sus objetivos y ambiciones. El foco es la persona como individuo único (citado en } \\
\text { Nadin, Orr y Meagher, 2013: 9) }\end{array}$ \\
\hline Sanderson (2000) & $\begin{array}{l}\text { Es un proceso de escucha y aprendizaje continuo, centrado en lo que importa ahora y no } \\
\text { en el futuro, y se centra en actuar en alianza con los familiares y amigos. No es sólo un con- } \\
\text { junto de técnicas nuevas que sustituyen a la programación individual. Se trata de una nueva } \\
\text { forma de trabajar con la persona con discapacidad, en la que es fundamental el compartir el } \\
\text { poder y la inclusión en la comunidad (citado en Nadin, Orr y Meagher, 2013: 9) }\end{array}$ \\
\hline $\begin{array}{l}\text { Dowling, Manthorpe } \\
\text { y Cowley (2007) }\end{array}$ & $\begin{array}{l}\text { Es el corazón de muchas políticas recientes en los servicios sociales. Hace referencia a un } \\
\text { enfoque en el que la persona planea su propio futuro y obtiene los servicios que necesita. } \\
\text { (citado en Nadin, Orr y Meagher, 2013: 9) }\end{array}$ \\
\hline $\begin{array}{l}\text { Mata y Carratalá } \\
\text { (2007) }\end{array}$ & $\begin{array}{l}{[\ldots \text {... proceso de colaboración para ayudar a las personas a acceder a los apoyos y servicios }} \\
\text { que necesitan para alcanzar una mayor calidad de vida basada en sus propias preferencias } \\
\text { y valores. Es un conjunto de estrategias para la planificación de la vida que se centra en las } \\
\text { elecciones y la visión de la persona y de su círculo de apoyo (p. 10) }\end{array}$ \\
\hline $\begin{array}{l}\text { A...] entendemos la PCP como un proceso de colaboración que pretende que la persona con } \\
\text { DI, junto con un grupo de apoyo, formule sus propios principios, planes y metas de futuro } \\
\text { (2016) plan de vida deseado), así como los medios, estrategias y acciones necesarias para conse- } \\
\text { guir sus logros (p. 197) }\end{array}$ \\
\hline
\end{tabular}

Fuente: Adaptado de Mata y Carratalá (2007), Nadin, Orr y Meagher (2013) y Arellano y Peralta (2016).

A pesar de que en cada una de las definiciones se hace hincapié en unos aspectos determinados, todas ellas ponen en común el principio más evidente de la PCP: el centro de las decisiones es la persona con discapacidad intelectual, a la cual hay que prestar los apoyos precisos para que consiga sus metas y objetivos $\mathrm{y}$, por ende, mejore su calidad de vida.

Uno de los aspectos más novedosos de la PCP en comparación con los métodos de carácter tradicional es que el énfasis no está en la carencia o ausencia de una determinada habilidad de la persona con discapacidad, sino que se hace hincapié en sus capacidades (Pallisera, 2011; Vatland, 2012). Otras características de esta metodología son las siguientes (Dowling et al., 2006; Serra, 2006; Mata y Carratalá, 2007; NSW Department of Ageing, Disability and Home Care, 2009; Pallisera, 2011; Stirk y Sanderson, 2012; Nadin et al., 2013):

- Implica cambiar la cultura de las organizaciones y sus jerarquías.

- Las organizaciones han de tener un sistema de valores y creencias compartidos por todos los implicados.

(C) Ediciones Universidad de Salamanca / CC BY-NC-ND

Siglo Cero, vol. 48 (2), n. ${ }^{\circ}$ 262, 2017, abril-junio, pp. 25-40 
EFECTOS Y BENEFICIOS DE LA PLANIFICACIÓN CENTRADA EN LA PERSONA (PCP) EN LAS PERSONAS ADULTAS CON DISCAPACIDAD INTELECTUAL, EN LAS ORGANIZACIONES Y EN LA COMUNIDAD

TANIA CUERVO RODRÍGUEZ, MARÍA TERESA IGLESIAS GARCÍA Y SAMUEL FERNÁNDEZ FERNÁNDEZ

- Los profesionales dejan de tener el rol de expertos, y pasan a actuar como educadores y facilitadores de aprendizaje.

- Tiene en cuenta los sueños, capacidades y deseos de la persona con discapacidad.

- Se favorece que la persona con discapacidad forme parte de entornos comunitarios inclusivos.

- La persona con discapacidad es empoderada a través de las elecciones.

- La persona con discapacidad es considerada como una persona con derechos.

- Las actividades que se realicen deben ser significativas para la persona.

- El proceso de PCP mantiene una perspectiva holística de la persona con discapacidad.

- Se produce una estrecha colaboración entre la persona, la familia y los profesionales.

- Los roles desempeñados por cada uno de los participantes son completamente respetados.

El objetivo de este estudio es analizar si realmente la Planificación Centrada en la Persona es efectiva cuando se aplica y, en este sentido, hemos desarrollado el procedimiento de búsqueda bibliográfica que se detalla a continuación, así como los resultados obtenidos y las conclusiones que de ellos se derivan.

\section{Procedimiento}

La búsqueda de artículos ha tenido lugar de mayo a julio de 2016. Se ha realizado a través de seis bases de datos: DIALNET, DOAJ, REDIB, ISOC, SCOPUS y ERIC. En cada una de ellas se han utilizado los descriptores "planificación centrada en la persona”, “pcp”, “person centred planning” y “person centered planning”, dando como resultado una gran cantidad de artículos, tal y como se indica en la Tabla 2.

Para la selección de los artículos que componen esta revisión bibliográfica se han seguido dos fases:

1. Lectura de los abstracts de los artículos obtenidos (2175) mediante el uso de los descriptores indicados para, de ese modo, hacer una preselección de los que más se acercan a la finalidad de esta revisión (205).

2. Establecimiento de criterios para la selección final. Estos han sido los siguientes:

- Ha de tratarse de investigaciones científicas y no de revisiones teóricas sobre la planificación centrada en la persona.

- Las investigaciones han de centrarse en alguno de los aspectos implicados en la implantación de la PCP y/o de los resultados obtenidos tras el uso de esta metodología.

- En las investigaciones han de venir expuestos los objetivos, instrumentos, el proceso y los resultados obtenidos.

- Estos estudios han de centrarse en la población con discapacidad intelectual (o múltiple) adulta, excluyendo aquellos que se centran en el uso de la PCP en la etapa escolar o posterior. 
EFECTOS Y BENEFICIOS DE LA PLANIFICACIÓN CENTRADA EN LA PERSONA (PCP) EN LAS PERSONAS ADULTAS CON DISCAPACIDAD INTELECTUAL, EN LAS ORGANIZACIONES Y EN LA COMUNIDAD TANIA CUERVO RODRÍGUEZ, MARÍA TERESA IGLESIAS GARCÍA Y SAMUEL FERNÁNDEZ FERNÁNDEZ

\begin{tabular}{|c|c|c|c|c|c|c|}
\hline & & $\begin{array}{l}\text { PLANIFICACIÓN } \\
\text { CENTRADA } \\
\text { EN LA PERSONA }\end{array}$ & $P C P$ & $\begin{array}{l}\text { PERSON CENTRED } \\
\text { PLANNING }\end{array}$ & $\begin{array}{c}\text { PERSON CENTERED } \\
\text { PLANNING }\end{array}$ & TOTAL \\
\hline \multirow[t]{3}{*}{ DIALNET } & Resultado & 152 & 129 & 63 & 6 & 350 \\
\hline & Fase 1 & 2 & 1 & 1 & 1 & 5 \\
\hline & \multicolumn{5}{|c|}{ Fase 2} & 1 \\
\hline \multirow[t]{3}{*}{$D O A J$} & Resultado & 0 & 367 & 10 & 10 & 387 \\
\hline & Fase 1 & 0 & 0 & 0 & 0 & 0 \\
\hline & \multicolumn{5}{|c|}{ Fase 2} & 0 \\
\hline \multirow[t]{3}{*}{ REDIB } & Resultado & 4 & 22 & 13 & 28 & 67 \\
\hline & Fase 1 & 0 & 0 & 0 & 0 & 0 \\
\hline & \multicolumn{5}{|c|}{ Fase 2} & 0 \\
\hline \multirow[t]{3}{*}{ ISOC } & Resultado & 24 & 34 & 0 & 0 & 58 \\
\hline & Fase 1 & 4 & 2 & 0 & 0 & 6 \\
\hline & \multicolumn{5}{|c|}{ Fase 2} & 2 \\
\hline \multirow[t]{3}{*}{ SCOPUS } & Resultado & 1 & 567 & 221 & 223 & 1.012 \\
\hline & Fase 1 & 0 & 6 & 41 & 57 & 104 \\
\hline & \multicolumn{5}{|c|}{ Fase 2} & 10 \\
\hline \multirow[t]{3}{*}{ ERIC } & Resultado & 8 & 114 & 41 & 138 & 301 \\
\hline & Fase 1 & 0 & 14 & 21 & 55 & 90 \\
\hline & \multicolumn{5}{|c|}{ Fase 2} & 5 \\
\hline \multirow[t]{3}{*}{ TOTAL } & Resultado & 189 & 1.233 & 348 & 405 & 2.175 \\
\hline & Fase 1 & 6 & 23 & 63 & 113 & 205 \\
\hline & \multicolumn{5}{|c|}{ Fase 2} & 18 \\
\hline
\end{tabular}

FUENTE: Elaboración propia.

En total se han seleccionado 18 artículos pertenecientes a 12 revistas de ámbito internacional y nacional (ver Tabla 3). En la Tabla 4 se muestran las características de cada uno de estos estudios, los cuales se realizan sobre grupos de diferente tamaño (oscilando $\mathrm{N}$ entre 3 y 93), persiguen diferentes objetivos y utilizan varios instrumentos de recogida de información, incluyendo tanto herramientas cualitativas como cuantitativas.

\section{TABLA 3. Revistas a las que pertenecen los artículos seleccionados}

\begin{tabular}{|l|c|c|}
\hline \multicolumn{1}{|c|}{ TítULO DE LA REVISTA } & ARTícUlOS & $\%$ \\
\hline American Journal on Mental Retardation & 3 & $16,66 \%$ \\
\hline British Journal of Learning Disabilities & 1 & $5,55 \%$ \\
\hline Contextos Educativos & 1 & $5,55 \%$ \\
\hline
\end{tabular}

(C) Ediciones Universidad de Salamanca / CC BY-NC-ND

Siglo Cero, vol. 48 (2), n. ${ }^{\circ}$ 262, 2017, abril-junio, pp. 25-40

$$
-29-
$$


EFECTOS Y BENEFICIOS DE LA PLANIFICACIÓN CENTRADA EN LA PERSONA (PCP) EN LAS PERSONAS ADULTAS CON DISCAPACIDAD INTELECTUAL, EN LAS ORGANIZACIONES Y EN LA COMUNIDAD TANIA CUERVO RODRÍGUEZ, MARÍA TERESA IGLESIAS GARCÍA Y SAMUEL FERNÁNDEZ FERNÁNDEZ

\begin{tabular}{|l|c|c|}
\hline \multicolumn{3}{|c|}{ TABLA 3. Revistas a las que pertenecen los artículos seleccionados } \\
\hline \multicolumn{1}{|c|}{ TíTULO DE LA REvisTA } & ARTículos & $\%$ \\
\hline Education and Training in Developmental Disabilities & 1 & $5,55 \%$ \\
\hline Health and Social Care in the Community & 1 & $5,55 \%$ \\
\hline Human Services Research and Development Center & 1 & $5,55 \%$ \\
\hline Journal of Applied Behavior Analysis & 2 & $11,11 \%$ \\
\hline Journal of Applied Research in Intellectual Disabilities & 2 & $11,11 \%$ \\
\hline Journal of Intellectual Disability Research & 1 & $11,11 \%$ \\
\hline Mental Retardation & 1 & $5,55 \%$ \\
\hline Research to Practice & 2 & $5,55 \%$ \\
\hline Siglo Cero & 18 & $11,11 \%$ \\
\hline
\end{tabular}

\begin{tabular}{|c|c|c|c|}
\hline \multicolumn{4}{|c|}{ TABLA 4. Características de los artículos seleccionados } \\
\hline & $\mathrm{N}$ & Овjetivos & $\begin{array}{c}\text { INSTRUMENTOS DE RECOGIDA } \\
\text { DE INFORMACIÓN }\end{array}$ \\
\hline $\begin{array}{l}\text { Human Services } \\
\text { Research and } \\
\text { Developmental } \\
\text { Center } \\
(1995)\end{array}$ & $\begin{array}{l}53 \text { personas con } \\
\text { discapacidad perte- } \\
\text { necientes a } 8 \text { organi- } \\
\text { zaciones. }\end{array}$ & $\begin{array}{l}\text { - Ofrecer a las personas un } \\
\text { futuro deseable. } \\
\text { - Incrementar relaciones in- } \\
\text { terpersonales, entornos or- } \\
\text { dinarios, hacer elecciones y } \\
\text { ser tratados con respeto. } \\
\text { - Ofrecer el apoyo necesario } \\
\text { para aumentar su compe- } \\
\text { tencia comunitaria. }\end{array}$ & $\begin{array}{l}\text { - Reuniones entre los profesionales de las } \\
\text { organizaciones participantes. }\end{array}$ \\
\hline $\begin{array}{l}\text { Stalker y } \\
\text { Campbell } \\
(1998)\end{array}$ & $\begin{array}{l}5 \text { organizaciones de } \\
\text { personas con dis- } \\
\text { capacidad. En cada } \\
\text { una se formaban } 20 \\
3 \text { personas en PCP. } \\
\end{array}$ & $\begin{array}{l}\text { - Evaluar la eficacia de la } \\
\text { PCP y su grado de éxito. } \\
\text { - Hacer recomendaciones } \\
\text { respecto a los planes de } \\
\text { futuro. }\end{array}$ & $\begin{array}{l}\text { - Entrevistas semiestructuradas a los parti- } \\
\text { cipantes que se formaban en PCP. }\end{array}$ \\
\hline $\begin{array}{l}\text { Whitney-Tomas } \\
\text { y Timmons } \\
\text { (1998) }\end{array}$ & $\begin{array}{l}10 \text { personas con dis- } \\
\text { capacidad. }\end{array}$ & $\begin{array}{l}\text { - Examinar el rol y la parti- } \\
\text { cipación de los facilitado- } \\
\text { res, familiares y amigos en } \\
\text { el proceso de PCP. }\end{array}$ & $\begin{array}{l}\text { - Entrevistas a personas con discapacidad, } \\
\text { familiares y facilitadores. } \\
\text { - Observación participante. }\end{array}$ \\
\hline $\begin{array}{l}\text { Reid, Everson y } \\
\text { Green (1999) }\end{array}$ & $\begin{array}{l}4 \text { personas con dis- } \\
\text { capacidad. }\end{array}$ & $\begin{array}{l}\text { - Evaluar la precisión de } \\
\text { un mapeo centrado en la } \\
\text { persona para identificar } \\
\text { las preferencias de las per- } \\
\text { sonas con múltiples y pro- } \\
\text { fundas discapacidades. }\end{array}$ & - Ítems de preferencia. \\
\hline
\end{tabular}


EFECTOS Y BENEFICIOS DE LA PLANIFICACIÓN CENTRADA EN LA PERSONA (PCP) EN LAS PERSONAS ADULTAS CON DISCAPACIDAD INTELECTUAL, EN LAS ORGANIZACIONES Y EN LA COMUNIDAD TANIA CUERVO RODRÍGUEZ, MARÍA TERESA IGLESIAS GARCÍA Y SAMUEL FERNÁNDEZ FERNÁNDEZ

\begin{tabular}{|c|c|c|c|}
\hline \multicolumn{4}{|c|}{ TABLA 4. Características de los artículos seleccionados } \\
\hline & $\mathrm{N}$ & Овjetivos & $\begin{array}{c}\text { INSTRUMENTOS DE RECOGIDA } \\
\text { DE INFORMACION }\end{array}$ \\
\hline $\begin{array}{l}\text { Green, } \\
\text { Middelton y } \\
\text { Reid (2000) }\end{array}$ & $\begin{array}{l}3 \text { personas con } \\
\text { discapacidad. }\end{array}$ & $\begin{array}{l}\text { - Evaluar las preferencias a } \\
\text { través de la PCP. } \\
\text { - Demostrar que los planes } \\
\text { centrados en la persona } \\
\text { son una buena opción } \\
\text { para evaluar las preferen- } \\
\text { cias. }\end{array}$ & - Planes personales. \\
\hline $\begin{array}{l}\text { Heller, Miller, } \\
\text { Hsieh y Sterns } \\
(2000)\end{array}$ & $\begin{array}{l}60 \text { personas con dis- } \\
\text { capacidad, divididos } \\
\text { en: } \\
-38 \text { grupo de inter- } \\
\quad \text { vención. } \\
-22 \text { grupo de com- } \\
\text { paración. }\end{array}$ & $\begin{array}{l}\text { - Examinar la efectividad de } \\
\text { un programa de PCP de } \\
\text { "Later-life planning" para } \\
\text { adultos con discapacidad. }\end{array}$ & $\begin{array}{l}\text { - The Inventory for Client and Agency } \\
\text { Planning. } \\
\text { - The Life Satisfaction Scale for Aging } \\
\text { Adults with Mental Retardation. } \\
\text { - Daily Choice Inventory. } \\
\text { - The Later-life Planning Curriculum Test. } \\
\text { - Entrevistas. }\end{array}$ \\
\hline $\begin{array}{l}\text { Holburn, } \\
\text { Jacobson, } \\
\text { Vietze, Schwartz } \\
\text { y Sersen } \\
(2000)\end{array}$ & $\begin{array}{l}37 \text { personas con dis- } \\
\text { capacidad, divididas } \\
\text { en: } \\
-20 \text { reciben PCP. } \\
\text { - } 17 \text { reciben planfi- } \\
\quad \text { cicaciones } \\
\text { tradicionales. }\end{array}$ & $\begin{array}{l}\text { - Crear un cuestionario que } \\
\text { cuantificara el proceso y } \\
\text { resultados de PCP. }\end{array}$ & - Cuestionario elaborado por los autores. \\
\hline $\begin{array}{l}\text { Menchetti y } \\
\text { García } \\
(2003)\end{array}$ & $\begin{array}{l}83 \text { personas con } \\
\text { discapacidad. }\end{array}$ & $\begin{array}{l}\text { - Examinar la herramienta } \\
\text { de PCP "Personal Career } \\
\text { Plan". } \\
\text { - Identificar las percepcio- } \\
\text { nes de los participantes. }\end{array}$ & $\begin{array}{l}\text { - Grupos focales con las personas con } \\
\text { discapacidad. }\end{array}$ \\
\hline $\begin{array}{l}\text { Combes, Hardy } \\
\text { y Buchan. } \\
(2004)\end{array}$ & $\begin{array}{l}2 \text { personas con } \\
\text { discapacidad. }\end{array}$ & $\begin{array}{l}\text { - Evaluar si la Q-methodo- } \\
\text { logy puede usarse con } \\
\text { personas con discapaci- } \\
\text { dad y su círculo de apoyo } \\
\text { para identificar las acti- } \\
\text { vidades que valoran para } \\
\text { hacer posteriores planifi- } \\
\text { caciones. }\end{array}$ & - Q-sort. \\
\hline $\begin{array}{l}\text { Holburn, } \\
\text { Jacobson, } \\
\text { Schwartz, Flory } \\
\text { y Vietze } \\
(2004)\end{array}$ & $\begin{array}{l}38 \text { personas con dis- } \\
\text { capacidad, divididos } \\
\text { en: } \\
\text { - } 20 \text { recibían PCP. } \\
\text { - } 20 \text { recibían plani- } \\
\quad \text { ficaciones } \\
\text { tradicionales. }\end{array}$ & \begin{tabular}{|l} 
- Determinar si la PCP \\
puede producir mejoras \\
en la calidad de vida de \\
las personas con discapa- \\
cidad. \\
- Conocer si la PCP pue- \\
de acelerar el proceso de \\
vida en la comunidad.
\end{tabular} & $\begin{array}{l}\text { - The Developmental Disabilities Profile } 2 . \\
\text { - The Personal Futures Planning } \\
\text { Indicators. } \\
\text { - The Indicators of Principles Scale. } \\
\text { - The Person-centered Planning Quality of } \\
\text { Life Indicators. }\end{array}$ \\
\hline
\end{tabular}


EFECTOS Y BENEFICIOS DE LA PLANIFICACIÓN CENTRADA EN LA PERSONA (PCP) EN LAS PERSONAS

ADULTAS CON DISCAPACIDAD INTELECTUAL, EN LAS ORGANIZACIONES Y EN LA COMUNIDAD

TANIA CUERVO RODRÍGUEZ, MARÍA TERESA IGLESIAS GARCÍA Y SAMUEL FERNÁNDEZ FERNÁNDEZ

\begin{tabular}{|c|c|c|c|}
\hline & $\mathrm{N}$ & OBjetivos & $\begin{array}{l}\text { INSTRUMENTOS DE RECOGIDA } \\
\text { DE INFORMACIÓN }\end{array}$ \\
\hline $\begin{array}{l}\text { Robertson et al. } \\
(2006 ; 2007 \mathrm{a}, \mathrm{b}, \\
\text { c; 2008) }\end{array}$ & $\begin{array}{l}93 \text { personas con } \\
\text { discapacidad. }\end{array}$ & $\begin{array}{l}\text { - Identificar el impacto y los } \\
\text { costes de la PCP. } \\
\text { - Identificar las barreras de } \\
\text { la puesta en práctica de la } \\
\text { PCP. } \\
\text { - Identificar qué factores } \\
\text { están asociados con la pro- } \\
\text { babilidad de recibir PCP y } \\
\text { con la mejora en las dife- } \\
\text { rentes áreas de la vida. } \\
\text { - Identificar el impacto y los } \\
\text { costes de la PCP. } \\
\end{array}$ & $\begin{array}{l}\text { - Health Survey for England. } \\
\text { - Index of Community Involvement. } \\
\text { - Social Network MAPS. } \\
\text { - Client Service Receipt Inventory. } \\
\text { - Strengths and Difficulties Questionnaire. } \\
\text { - Adaptación de la Risk Scale. } \\
\text { - ABS. } \\
\text { - PAS-ADD Checklist. } \\
\text { - Learning Disabilities Casemix Scale. } \\
\text { - Client Service Receipt Inventory. } \\
\text { - Residencial Services Setting Questionnaire. } \\
\text { - Historial residencial. } \\
\text { - Actividades de PCP. } \\
\text { - Información y materiales de auditoría. } \\
\text { - Social Network Map. } \\
\text { - Questionnaire. } \\
\text { - Risks Scale. } \\
\text { - Psychiatric Assesment Schedule for } \\
\text { Adults with Developmental Disabilities } \\
\text { Checklist. } \\
\text { - English Index of Multiple Deprivation. } \\
\text { - Learning Disabilities Casemix Scale. } \\
\text { - Adaptive Behavior Scale. } \\
\text { - Facilitator Questionnaire. }\end{array}$ \\
\hline $\begin{array}{l}\text { Espiner y } \\
\text { Harnett } \\
(2011)\end{array}$ & $\begin{array}{l}10 \text { personas con } \\
\text { discapacidad. }\end{array}$ & $\begin{array}{l}\text { - Comprender los ele- } \\
\text { mentos que sustentan el } \\
\text { enfoque de la PCP en la } \\
\text { realización de planes per- } \\
\text { sonales. }\end{array}$ & $\begin{array}{l}\text { - Entrevistas individualizadas. } \\
\text { - Cuestionario de escala Likert elaborado } \\
\text { por los autores. }\end{array}$ \\
\hline $\begin{array}{l}\text { Hillman et al. } \\
\text { (2012) }\end{array}$ & $\begin{array}{l}9 \text { personas con } \\
\text { discapacidad. }\end{array}$ & \begin{tabular}{|l|} 
- Conocer la relación entre \\
los derechos y las dinámi- \\
cas de PCP.
\end{tabular} & $\begin{array}{l}\text { - Entrevistas. } \\
\text { - Observaciones participantes. }\end{array}$ \\
\hline $\begin{array}{l}\text { Arellano y } \\
\text { Peralta } \\
(2016)\end{array}$ & 18 profesionales. & $\begin{array}{l}\text { - Comprobar si los profe- } \\
\text { sionales conocen en qué } \\
\text { consiste la PCP, si han re- } \\
\text { cibido formación. } \\
\text { - Identificar las dificultades } \\
\text { para implantar PCP. }\end{array}$ & $\begin{array}{l}\text { - Cuestionario ad hoc elaborado por los } \\
\text { autores. }\end{array}$ \\
\hline
\end{tabular}

FUENTE: Elaboración propia.

Como se observa en la Tabla 4, de los 18 artículos seleccionados, el 55,55\% tiene una muestra inferior a 50 participantes, y el $94,44 \%$ se trata de estudios anglosajones, siendo la única excepción el estudio de Arellano y Peralta (2016), que ha sido elaborado en contexto español.

(C) Ediciones Universidad de Salamanca / CC BY-NC-ND

Siglo Cero, vol. 48 (2), n. ${ }^{\circ}$ 262, 2017, abril-junio, pp. 25-40 
EFECTOS Y BENEFICIOS DE LA PLANIFICACIÓN CENTRADA EN LA PERSONA (PCP) EN LAS PERSONAS ADULTAS CON DISCAPACIDAD INTELECTUAL, EN LAS ORGANIZACIONES Y EN LA COMUNIDAD

TANIA CUERVO RODRÍGUEZ, MARÍA TERESA IGLESIAS GARCÍA Y SAMUEL FERNÁNDEZ FERNÁNDEZ

\section{Resultados}

A continuación se muestran los resultados obtenidos tras el análisis de los 18 estudios sobre la PCP. Siguiendo a Miles y Huberman (1994), se organiza una matriz de categorías con las principales aportaciones: el impacto que tiene esta metodología directamente en la persona con discapacidad, en las organizaciones y en la comunidad en la que se encuentran estas personas. En cada una de ellas se ha registrado la frecuencia de los elementos de mayor significado para los autores/as. Además, se incluye un último apartado de las principales barreras identificadas a la hora de implantar la PCP.

\subsection{En la vida diaria de las personas con discapacidad intelectual}

Para que la PCP tenga un impacto significativo en la vida de las personas con discapacidad intelectual, los integrantes del grupo de apoyo han de involucrarse, apoyar y proporcionar los apoyos que requiera la persona. Whitney-Tomas y Timmons (1998) identifican una serie de factores que fomentan que la PCP se desarrolle de una forma adecuada: "hablar directamente con la persona", "verificar y/o comprobar el significado de sus respuestas y sugerencias”, "hablarle al mismo nivel de lenguaje”, "esperar su respuesta y no adelantarse", "permitir que cambie de tema”, "mantener un tono positivo" y "dejar de lado los miedos".

En relación con esto, Combes et al. (2004) informan que las personas de un círculo de apoyo son capaces de representar correctamente a las personas con discapacidad, ya que encontraron unas correlaciones altas al respecto. Las personas con discapacidad, por su parte, han reportado que lo que más valoran es estar con la familia y amigos, mientras que lo que menos son las tareas y actividades de limpieza.

Las redes de apoyo de la investigación elaborada por Hillman et al. (2012) mostraron la importancia de formar a las personas con discapacidad en la defensa de sus derechos y de que sean el centro de todas las decisiones, deseando para ellos: felicidad y seguridad, ser respetados, que tengan buenas relaciones, una mayor autonomía, que sean miembros de la comunidad y, en general, que disfruten de la vida.

Por otro lado, Espiner y Harnett (2011) señalan que 9 de cada 10 adultos indicaron que el facilitador había creado un ambiente en el que se sentían escuchados. A su vez, 7 de cada 10 se mostraron contentos con su plan, 2 no hablaron de la efectividad del mismo y 1 se mostraba frustrado al no lograr las metas fijadas en el documento.

En la investigación sobre las preferencias realizada por Reid et al. (1999), se ha observado que, de los 24 ítems y actividades estimados como preferentes en los planes centrados en la persona, el $33 \%$ de ellos se consideran con un alto nivel de preferencia y el $42 \%$ como preferencia moderada. Por lo tanto, la mayoría de las actividades incluidas en estos planes resultan satisfactorias para las personas con discapacidad participantes. Por otra parte, coinciden con Green et al. (2000) al remarcar la importancia de evaluar sistemáticamente las preferencias de las personas.

Holburn et al. (2004) señalaron que 18 de las 19 personas que componían el grupo de intervención (el 94,7\%) se han movido a la comunidad, mientras que, en el grupo de 
EFECTOS Y BENEFICIOS DE LA PLANIFICACIÓN CENTRADA EN LA PERSONA (PCP) EN LAS PERSONAS ADULTAS CON DISCAPACIDAD INTELECTUAL, EN LAS ORGANIZACIONES Y EN LA COMUNIDAD TANIA CUERVO RODRÍGUEZ, MARÍA TERESA IGLESIAS GARCÍA Y SAMUEL FERNÁNDEZ FERNÁNDEZ

control, sólo 5 de los 18 participantes (el 27,7\%) han salido de la institución. Los autores han llegado a la conclusión de que la PCP produce más resultados positivos a nivel de calidad de vida y de movimiento a la comunidad en comparación con los enfoques más tradicionales, coincidiendo con lo reportado por los participantes de la investigación de Hillman et al. (2012).

En el estudio de Menchetti y García (2003), mediante la aplicación del Personal Career Plan se ha conseguido que el 58\% de la muestra obtuviera un empleo en un ámbito y una ubicación idénticos a lo expresado. El 29\% lograron un empleo en una ocupación y una ubicación relacionadas con lo expresado, mientras que el 13\% obtuvo un empleo en una ocupación y una ubicación no relacionadas con lo expresado.

Otro modelo de PCP, el llamado Later-life Planning, ha sido estudiado por Heller et al. (2000), llegando a la conclusión de que, tras su implementación, las personas del grupo de intervención han incrementado significativamente el número de elecciones realizadas respecto al grupo de comparación. A pesar de ello, no se han producido cambios significativos en la satisfacción con la vida. A su vez, cada uno de los participantes ha conseguido realizar 2,7 metas, realizándose durante toda la investigación un total de 86. Las metas que más se han producido han sido en las áreas de "actividades sociales y recreativas", "mantener o cambiar la situación laboral”, "mantener o cambiar la situación en casa” y "mejorar la salud y el bienestar".

En la investigación realizada por Robertson et al. (2007a) se llegó a las siguientes conclusiones:

a) Aquellas personas que tienen problemas de salud mental, emocional y/o conductual presentan menos probabilidades de tener un plan, y si llegaban a tenerlo, sus opciones de tener éxito en áreas como "tamaño de las redes sociales", “contacto con amigos" y "horas y número de actividades” eran menores.

b) Tener movilidad reducida también hace menos probable que reciban un plan, pero cuando lo tenían se beneficiaban más de las áreas de "contacto con la familia”, "horas semanales de actividades programadas" y "número de actividades en la comunidad”.

c) Los que tuviesen autismo es probable que no llegasen a tener un plan.

d) Las áreas en las que más se benefician las mujeres son "número de actividades en la comunidad" y "elecciones".

e) Las áreas en las que más se benefician los hombres son "número de horas semanales de actividades programadas" y "contacto con los amigos”.

El artículo publicado por Robertson et al. (2008), que también hace referencia al mismo estudio, concluyó que los cambios significativamente estadísticos en las personas con discapacidad han sido:

- El tamaño de las redes sociales aumentó un $52 \%$.

- Había una probabilidad 2,4 veces mayor de tener contacto con la familia.

- El número de actividades aumentó un $40 \%$ y su variedad un $25 \%$.

- Las horas semanales de actividades aumentaron un 33\%.

- Se produjeron 2,8 veces más probabilidades de realizar elecciones. 
EFECTOS Y BENEFICIOS DE LA PLANIFICACIÓN CENTRADA EN LA PERSONA (PCP) EN LAS PERSONAS ADULTAS CON DISCAPACIDAD INTELECTUAL, EN LAS ORGANIZACIONES Y EN LA COMUNIDAD TANIA CUERVO RODRÍGUEZ, MARÍA TERESA IGLESIAS GARCÍA Y SAMUEL FERNÁNDEZ FERNÁNDEZ

Pero no todos los cambios producidos fueron estadísticamente significativos, destacando como no significativos el aumento de un $17 \%$ de contacto con familiares; un $19 \%$ el número de visitas a, de o con familia, y del $28 \%$ en el número de visitas a, de o con amigos. Tampoco ha sido significativo el aumento en las probabilidades (un $2,0)$ de haber tenido contacto activo con miembros de la familia ni el aumento en las oportunidades (un 1,8) de tener actividades recreativas programadas.

Es destacable también que no todos los cambios hayan sido positivos, pues también se produjeron aumentos no significativos en la subescala de problemas emocionales (59\%), en el número de problemas de salud informados (67\%) y en la puntuación de impacto (58\%) y descensos no significativos en la subescala prosocial (14\%).

Por su parte, los datos de la misma investigación publicados por Robertson et al. (2007b) muestran que las mejoras en las relaciones sociales se asocian con tener un pequeño número de relaciones al comienzo del estudio y vivir en áreas que tengan poca afluencia. Las mejoras en las actividades diarias se asocian con tener bajas puntuaciones en las escalas de comportamiento y tener pocas actividades al comienzo. El incremento del contacto con amigos está relacionado con tener más problemas de salud que otros participantes, tener puntuaciones bajas en la subescala de hiperactividad y altas en la de proactividad. El aumento del contacto con familiares está asociado, no significativamente, con la subescala de problemas con sus pares. Y, por último, el incremento en las elecciones se relaciona con el informe de los facilitadores sobre las barreras que se producen a la hora de implantar la PCP.

En resumen, Robertson et al. (2008 y 2007b) concluyen que la PCP tiene efectividad en cuatro áreas: "participación en la comunidad", "contacto con los amigos", "contacto con la familia" y "elección”.

\subsection{En las organizaciones}

En el estudio más actual, desarrollado por Arellano y Peralta (2016), se ha podido observar la falta de formación e información que tienen los profesionales sobre esta metodología. El 39\% manifiesta no haber tenido ningún tipo de formación en PCP, mientras que el $44 \%$ considera que tienen poco tiempo para llevarla a cabo y el $44 \%$ de los participantes describen la PCP como un plan de atención individualizado. A pesar de que el $100 \%$ considera que la PCP facilita una inserción social, que el $78 \%$ cree importante conocer las necesidades de las persona y que la mayoría está de acuerdo en que la autodeterminación es un factor clave para su desarrollo, el 33\% manifiesta que la PCP puede realizarse sin que la persona con discapacidad conozca el proceso que va a llevar a cabo, actitud que no concuerda con los principios de esta metodología.

Las organizaciones participantes en el estudio de Human Services Research and Development Center (1995) han sido capaces de llevar a cabo cambios más acordes con los enfoques y valores de la Planificación Centrada en la Persona, teniendo cierto éxito en todo el sistema de apoyos en el que participan las personas con discapacidad. Además, 3 de estas instituciones han llegado incluso a crear grupos de autogestores en los que los usuarios del servicio pueden debatir sobre temas de su interés. 
EFECTOS Y BENEFICIOS DE LA PLANIFICACIÓN CENTRADA EN LA PERSONA (PCP) EN LAS PERSONAS ADULTAS CON DISCAPACIDAD INTELECTUAL, EN LAS ORGANIZACIONES Y EN LA COMUNIDAD TANIA CUERVO RODRÍGUEZ, MARÍA TERESA IGLESIAS GARCÍA Y SAMUEL FERNÁNDEZ FERNÁNDEZ

Otros estudios, como el de Espiner y Harnett (2011) y Stalker y Campbell (1998), hacen breves referencias a los efectos de la PCP sobre la propia organización. En el primero de ellos, un profesional de los que participaban en la investigación ha expresado que es necesario involucrar a más familiares y miembros de la comunidad en el proceso de PCP. En el segundo estudio, se ha visto que el director tenía poca formación en planificación centrada en la persona, pero que durante el proceso daba la responsabilidad a los participantes para que "hiciesen las cosas por sí mismos".

Holburn et al. (2000) tenían como objetivo realizar un cuestionario que cuantificara el proceso de PCP, el cual han conseguido desarrollar con una sólida consistencia interna y con una razonable y positiva asociación entre los procesos y resultados de esta metodología.

Por último, en la investigación realizada por Robertson et al. (2008) en Reino Unido, se han calculado los costes directos e indirectos de la introducción de la PCP. En cuanto a los costes directos, se ha visto que por cada participante el coste es de $1.202 \$$ (calculado para $\mathrm{n}=93$ ), mientras que el coste calculado para $\mathrm{n}=65$ (los que tuvieron un plan) es de 1.718. Respecto a los costes indirectos, el total de los costes antes de la PCP por participante (según $n=93$ ) era de $2.421 \$$. Estas cifras sufrieron un aumento, no significativo, de un $2,2 \%$, habiendo crecido hasta $2.476 \$$.

\subsection{En la comunidad}

Pocas han sido las referencias a los beneficios que se han producido en la comunidad en la que viven las personas con discapacidad que participan en un proceso de PCP. Únicamente en el estudio de Human Services Research and Development Center (1995) hacen alusión a que, debido a los cambios producidos en las organizaciones, los individuos empezaban a tener un rol integrado en las comunidades, mostrando sus integrantes una actitud positiva en muchas de las situaciones que han ido produciéndose.

\subsection{Barreras en la implantación de la PCP}

Debido a la gran cantidad de factores que intervienen en la implantación de una metodología como la planificación centrada en la persona, no es de extrañar que surjan una serie de impedimentos en su correcto desarrollo.

En el estudio de Robertson et al. (2007c) las personas con discapacidad han valorado que la principal barrera es "la reticencia de la comunidad de dar su tiempo y apoyo", con un $27 \%$ de puntuación total, seguida de: "servicios de día limitados" $(25 \%)$, "alojamientos limitados" (24\%) y "esperar listas de espera para los servicios" $(24 \%)$. En cambio, han otorgado menos puntuaciones a las barreras de "lo que el participante quiere" $(2 \%)$ y "metas no realistas" (5\%).

En este mismo artículo se identifican las principales barreras organizacionales, siendo "falta de fondos" (40\%), "falta de formación y facilitadores comprometidos" 
EFECTOS Y BENEFICIOS DE LA PLANIFICACIÓN CENTRADA EN LA PERSONA (PCP) EN LAS PERSONAS ADULTAS CON DISCAPACIDAD INTELECTUAL, EN LAS ORGANIZACIONES Y EN LA COMUNIDAD TANIA CUERVO RODRÍGUEZ, MARÍA TERESA IGLESIAS GARCÍA Y SAMUEL FERNÁNDEZ FERNÁNDEZ

(38\%) y “falta de formación en PCP” (37\%) las que más puntuaciones han obtenido. La barrera menos valorada ha sido la de "falta de compromiso de los servicios con la PCP” (19\%).

A su vez, y en general, las principales barreras en la implementación de la planificación centrada en la persona en el estudio de Robertson et al. (2007c) han sido "falta de tiempo del personal para trabajar en PCP" (49\%), "escasez de personal” (40\%) y "dificultades prácticas inherentes a la organización de reuniones de planificación” (35\%).

Roberston et al. (2006 y 2007a) asocian al fracaso de la puesta en práctica de la PCP las "metas que no se cumplen", "metas limitadas a opciones previamente disponibles para los usuarios de los servicios", "fracaso en atender a metas en áreas más ambiciosas o controvertidas como la sexualidad" y "metas que no corresponden con las preferencias evaluadas por vías alternativas".

En su artículo, Menchetti y García (2003) manifiestan los impedimentos con los que se encontraron a la hora de desarrollar el "Personal Career Plan": "dificultad para que las personas expresen su visión”, "impedimentos sistemáticos ya que a veces la PCP no es práctica", "estrés personal" y "grupos de apoyo poco flexibles a la hora de adaptarse a los roles y metas de las personas con discapacidad”.

Por su parte, Heller et al. (2000) han identificado en su estudio las siguientes barreras: "limitaciones físicas y cognitivas", "problemas de salud", "no recibir un adecuado apoyo por parte de la familia o del personal", "inadecuadas opciones residenciales o de trabajo" y "problemas en el transporte".

\section{Conclusiones}

A la vista de los resultados hallados mediante esta revisión bibliográfica, se ha llegado a las siguientes conclusiones:

1. Es indudable que la Planificación Centrada en la Persona tiene beneficios en la vida de las personas con discapacidad intelectual en diferentes áreas. El problema se presenta cuando las propias organizaciones o entidades no son capaces de romper la jerarquía tradicional de los servicios, pasando a desempeñar los profesionales su rol al mismo nivel que el resto del círculo de apoyo. La PCP precisa que las personas tengan un espíritu de cooperación, que se antepongan los intereses de la persona con discapacidad ante los de la propia organización. El grupo de apoyo ejerce un papel muy importante en el desarrollo de la PCP. Gracias a él es posible que la persona consiga sus metas establecidas. En el apartado de resultados se expone cómo Whitney-Tomas y Timmons (1998) han proporcionado una serie de pautas para el correcto devenir de estos grupos, los cuales se complementan con un dato proporcionado por Hillman et al. (2012), que reconocen que la escucha a la persona con discapacidad es un aspecto muy importante ya que muestra respeto y aprecio. A su vez, este grupo ha de ser capaz de guiar a la persona con discapacidad para que se fije unos objetivos reales acordes con su situación personal, no exagerados, sin llegar a ejercer un papel sobreprotector sobre él/ella. Con ello se evitarán los

(c) Ediciones Universidad de Salamanca / CC BY-NC-ND

Siglo Cero, vol. 48 (2), n. ${ }^{\circ}$ 262, 2017, abril-junio, pp. 25-40

$$
-37-
$$


EFECTOS Y BENEFICIOS DE LA PLANIFICACIÓN CENTRADA EN LA PERSONA (PCP) EN LAS PERSONAS ADULTAS CON DISCAPACIDAD INTELECTUAL, EN LAS ORGANIZACIONES Y EN LA COMUNIDAD

TANIA CUERVO RODRÍGUEZ, MARÍA TERESA IGLESIAS GARCÍA Y SAMUEL FERNÁNDEZ FERNÁNDEZ

sentimientos de frustración y los cambios no positivos mostrados en el estudio de Robertson et al. (2008): problemas emocionales, problemas de salud, descenso en la puntuación de la subescala prosocial...

2. Sorprendente es un dato aportado por Arellano y Peralta (2016), en el cual se muestra que el $33 \%$ de los profesionales que participaron en el estudio consideran que "[...] la PCP puede desarrollarse sin que la persona conozca el proceso que va a llevar a cabo [...]” (pp. 204-205). Si la finalidad de la Planificación Centrada en la Persona es que esta tenga voz en todas las decisiones... ¿ ¿ómo es posible que los profesionales mantengan esta idea? Esto demuestra la falta de formación e información que aún en tiempos actuales sigue existiendo en nuestros centros sobre esta metodología.

3. La PCP tiene una serie de beneficios en la vida de la persona con discapacidad intelectual: se produce un aumento del tamaño de las redes sociales (tanto con familiares como con amigos), se incrementa el número de actividades que realiza, así como su variedad y se da una mayor probabilidad de que la persona con discapacidad realice elecciones.

Aunque la PCP produce efectos positivos, no se puede caer en la falsa idea de que sólo por utilizar esta metodología ya se consiguen los objetivos fijados, al contrario, han de llevarse a cabo otras actuaciones como: análisis de las necesidades de apoyo de la persona con discapacidad (mediante escalas específicas como la SIS), actividades de sensibilización comunitarias, promoción de entornos inclusivos...

Los resultados y las conclusiones expuestas en este estado de la cuestión coinciden con los hallazgos encontrados por Claes et al. (2010) en su revisión bibliográfica, la cual se compone de 15 artículos.

Por último, aunque existe mucha literatura sobre la PCP de carácter anecdótico, estudios de caso o estudios cualitativos (Rasheed, Fore y Miller, 2006), son pocas las investigaciones en las que se realiza una investigación a fondo sobre su implantación, sobre su desarrollo y/o sobre sus resultados, por lo que no ha sido posible realizar un estudio metaanalítico ya que no todos los trabajos nos ofrecen información objetiva sobre el tamaño de efecto conseguido con las intervenciones. Además, el 55,55\% de las investigaciones analizadas tienen una muestra inferior a 50 participantes, por lo que puede ser difícil extrapolar sus resultados.

\section{Referencias bibliográficas}

Arellano, A. y Peralta, F. (2016). La planificación centrada en la persona: un ejemplo de buena práctica en el ámbito de la discapacidad intelectual. Contextos Educativos, 19, 195212. Recuperado el 7 de junio de 2016, de https://publicaciones.unirioja.es/ojs/index.php/ contextos/article/download/2754/2675.

Carbonell, E. (2006). Planificación centrada en la persona y discapacidad: barreras y oportunidades de mejora en Cataluña. Revista Médica Internacional Sobre el Síndrome de Down, $10(1), 13-16$. 
EFECTOS Y BENEFICIOS DE LA PLANIFICACIÓN CENTRADA EN LA PERSONA (PCP) EN LAS PERSONAS ADULTAS CON DISCAPACIDAD INTELECTUAL, EN LAS ORGANIZACIONES Y EN LA COMUNIDAD TANIA CUERVO RODRÍGUEZ, MARÍA TERESA IGLESIAS GARCÍA Y SAMUEL FERNÁNDEZ FERNÁNDEZ

Claes, C., Hove, G., Vandevelde, S., Loon, J. y Schalock, R. (2010). Person- centered planning: analysis of research and effectiveness. Intellectual and Developmental Disabilities, 48 (6), 432-453.

Cole, A., McIntosh, B. y WhitTaker, A. (2000). We want our voices heard developing new lifestyles with disabled People. Bristol: Policy Press.

Combes, H., Hardy, G. y Buchan, L. (2004). Using Q methodology to involve people with intellectual disability in evaluating person-centred planning. Journal of Applied Research in Intellectual Disabilities, 17, 149-159.

Davis, C., Cornman, C., Lane, M. y Patton, M. (2005). Person-centered planning for consumer-directed care for the elderly and disabled. Care Management Journals, 6 (3), 122-130.

Dowling, S., Manthorpe, J. y Cowley, S. (2007). Working on Person-centred planning: From Amber to Green Light. Journal of Intellectual Disabilities, 11 (1), 65-82.

Dowling, S., Manthorpe, J., Cowley, S., King, S., Raymond, V., Perez, W. et al. (2006). Person-centred planning in social care. A scoping review. Recuperado el 30 de junio de 2016, de https://www.jrf.org.uk/sites/default/files/jrf/migrated/files/9781859354803.pdf.

ESPINER, D. y HARNETT, F. (2011). "I felt i was in control of the meeting": facilitating planning with adults with an intellectual disability. British Journal of Learning Disabilities, 40, 62-70.

Flannery, K., Newton, S., Horner, R., Slovic, R., Blumberg, R. y Ard, W. (2000). The impact of person centered planning on the content and organization of individual supports. Career Development for Exceptional Individuals, 23, 123-137.

Green, C., Middelton, S. y Reid, D. (2000). Embedded evaluation of preferences sampled from person-centered plans for people with profound multiple disabilities. Journal of Applied Behavior Analysis, 33 (4), 639-642.

Heller, T., Miller, A., Hsieh, K. y Sterns, H. (2000). Later-life planning promoting knowledge of options and choice-making. Mental Retardation, 38 (5), 395-406.

Hillman, A., Donelly, M., Whitaker, L., Dew, A., Stancliffe, R., Knox, M. et al. (2012). Experiencing rights within positive, person-centred support networks of people with intellectual disability in Australia. Journal of Intellectual Disability Research, 56 (2), 1065-1075.

Holburn, S., Jacobson, J., Schwartz, A., Flory, M. y Vietze, P. (2004). The Willowbrook Futures Project: a longitudinal analysis of person-centered planning. American Journal on Mental Retardation, 109 (1), 63-76.

Holburn, S., Jacobson, J., Vietze, P., Schwartz, A. y Sersen, E. (2000). Quantifying the process and outcomes of person-centered planning. American Journal on Mental Retardation, 105 (5), 402-416.

Human Services Research and Developmental Center (1995). Person-centered agency design: a three year project 1991-1994. Recuperado el 16 de junio de 2016, de http://files.eric. ed.gov/fulltext/ED400652.pdf.

LunT, J. y Hinz, A. (2014). Formación y práctica de la PCP. Una perspectiva Europea: experiencias del proyecto "Nuevos Caminos Hacia la Inclusión". Madrid: FEAPS.

Mata, G. y Carratalá, A. (2007). Planificación centrada en la persona. Madrid: FEAPS. Recuperado el 30 de junio, de http://www.feaps.org/archivo/publicaciones-feaps/libros/cuadernos-de-buenas-practicas/123-planificacion-centrada-en-la-persona-.html.

Menchetti, B. y García, L. (2003). Personal and employment outcomes of person-centered career planning. Education and Training in Developmental Disabilities, 38 (2), 145-156.

Miles, M. y Huberman, A. (1994). Data management and analysis methods. En N. Denzin e Y. Lincoln (Eds.), Handbook of qualitative research (pp. 429-444). London: Sage Publication.

Nadin, P., Orr, M. y Meagher, J. (2013). Good practice guidelines for a person-centred planning and goal setting for people with psychosocial disability. Recuperado el 19 de junio

(C) Ediciones Universidad de Salamanca / CC BY-NC-ND

Siglo Cero, vol. 48 (2), n. ${ }^{\circ}$ 262, 2017, abril-junio, pp. 25-40

$$
-39-
$$


EFECTOS Y BENEFICIOS DE LA PLANIFICACIÓN CENTRADA EN LA PERSONA (PCP) EN LAS PERSONAS ADULTAS CON DISCAPACIDAD INTELECTUAL, EN LAS ORGANIZACIONES Y EN LA COMUNIDAD TANIA CUERVO RODRÍGUEZ, MARÍA TERESA IGLESIAS GARCÍA Y SAMUEL FERNÁNDEZ FERNÁNDEZ

de 2016, de http://docplayer.net/355719-Good-practice-guidelines-for-person-centredplanning-and-goal-setting-for-people-with-psychosocial-disability.html.

NSW Department of Ageing, Disability and Home Care (2009). Exploring an implementing person centred approaches. Recuperado el 29 de junio, de https://www.adhc.nsw.gov. au/_data/assets/file/0005/228290/DADHC_PersonCentred201208.pdf.

PAllisera, M. (2011). La planificación centrada en la persona (PCP): una vía para la construcción de proyectos personalizados con personas con discapacidad intelectual. Revista Iberoamericana de Educación, 56 (3). Recuperado el 17 de julio de 2016, de http://rieoei.org/ deloslectores/4092Pallisera.pdf.

Rasheed, S., Fore, C. y Miller, S. (2006). Person-centered planning: practices, promises and provisos. The Journal for Vocational Special Needs Education, 28 (3), 47-59.

Reid, D., Everson, J. y Green, C. (1999). A systematic evaluation of preferences identified through person-centered planning for people with profound multiple disabilities. Journal of Applied Behavior Analysis, 32 (4), 467-477.

Robertson, J., Emerson, E., Elliott, J. y Hatton, C. (2007). El impacto de la planificación centrada en la persona en las personas con discapacidad intelectual en Inglaterra: Un resumen de hallazgos. Siglo Cero, 38 (223), 5-24.

Robertson, J., Emerson, E., Hatton, C., Elliott, J., McIntosh, B., Swift, P. et al. (2006). Longitudinal analysis of the impact and cost of person-centered planning for people with intellectual disabilities in England. American Journal on Mental Retardation, 111 (6), 400-416.

Robertson, J., Emerson, E., Hatton, C., Elliott, J., Mcintosh, B., Swift, P. et al. (2007). Person-centred planning: factors associated with successful outcomes for people with intellectual disabilities. Journal of Intellectual Disability Research, 51 (3), 232-243.

Robertson, J., Emerson, E., Hatton, C., Elliott, J., McIntosh, B., Swift, P. et al. (2008). Análisis longitudinal del impacto y coste de la planificación centrada en la persona para personas con discapacidad intelectual en Inglaterra. Siglo Cero, 39 (225), 5-30.

Robertson, J., Hatton, C., Emerson, E., Elliott, J., Mcintosh, B., Swift, P. et al. (2007). Reported barriers to the implementation of person-centred planning for people with intellectual disabilities in the UK. Journal of Applied Research in Intellectual Disabilities, 20, 297-307.

SANDERson, H. (2000). Person-centred planning: key features and approaches. Joseph Roundtree Foundation. Recuperado el 20 de julio de 2007, de http://www.familiesleadingplanning.co.uk/documents/pcp \%20key\%20features $\% 20$ and $\% 20$ styles.pdf.

SERRA, F. (2006). Alternativas para el desarrollo de procesos personales de futuro para las personas con discapacidades significativas. Siglo Cero, 37 (229), 59-74.

Stalker, K. y Campbell, V. (1998). Person centred planning: an evaluation of a training programme. Health and Social Care in the Community, 6 (2), 130-134.

STIRK, S. y SANDERSON, H. (2012). Creating person-centred organizations. Strategies and tools for managing change in health, social care and the voluntary sector. London: Jessica Kingsley Pub.

VATLAND, C. (2012). The effect of structured team meetings and performance feedback on person-centered planning activities (tesis doctoral). Department of Special Education and Clinical Sciences, University of Oregon.

Whitney-Tomas, J. y Timmons, J. (1998). Building authentic visions: how to support the focus person in person centered planning. Research to Practice, 4 (3), 3-4.

(C) Ediciones Universidad de Salamanca / CC BY-NC-ND

Siglo Cero, vol. 48 (2), n. ${ }^{\circ}$ 262, 2017, abril-junio, pp. 25-40 